\title{
MYC Expression and Metabolic Redox Changes in Cancer Cells: A Synergy Able to Induce Chemoresistance
}

\author{
Barbara Marengo $\left(\mathbb{D},{ }^{1}\right.$ Ombretta Garbarino $\left(\mathbb{D},{ }^{1}\right.$ Andrea Speciale, ${ }^{2}$ Lorenzo Monteleone, ${ }^{1}$ \\ Nicola Traverso $\mathbb{D}^{1},{ }^{1}$ and Cinzia Domenicotti $\mathbb{D D}^{1}$ \\ ${ }^{1}$ Department of Experimental Medicine, General Pathology Section, University of Genova, Italy \\ ${ }^{2}$ UOC Mutagenesis and Oncologic Prevention, Ospedale Policlinico San Martino, Genova, Italy \\ Correspondence should be addressed to Barbara Marengo; barbara.marengo@unige.it
}

Received 8 April 2019; Revised 10 June 2019; Accepted 17 June 2019; Published 25 June 2019

Academic Editor: Alexandros Georgakilas

Copyright (c) 2019 Barbara Marengo et al. This is an open access article distributed under the Creative Commons Attribution License, which permits unrestricted use, distribution, and reproduction in any medium, provided the original work is properly cited.

\begin{abstract}
Chemoresistance is due to multiple factors including the induction of a metabolic adaptation of tumor cells. In fact, in these cells, stress conditions induced by therapies stimulate a metabolic reprogramming which involves the strengthening of various pathways such as glycolysis, glutaminolysis and the pentose phosphate pathway. This metabolic reprogramming is the result of a complex network of mechanisms that, through the activation of oncogenes (i.e., MYC, HIF1, and PI3K) or the downregulation of tumor suppressors (i.e., TP53), induces an increased expression of glucose and/or glutamine transporters and of glycolytic enzymes. Therefore, in order to overcome chemoresistance, it is necessary to develop combined therapies which are able to selectively and simultaneously act on the multiple molecular targets responsible for this adaptation. This review is focused on highlighting the role of MYC in modulating the epigenetic redox changes which are crucial in the acquisition of therapy resistance.
\end{abstract}

\section{Cancer Metabolic Reprogramming}

Metabolic reprogramming is an early event in the carcinogenic process, and it is involved in the development of malignancy and the acquisition of most cancer hallmarks [1]. The first metabolic phenotype observed in cancer cells was described by Otto Warburg, a German biochemist, as a shift from oxidative phosphorylation (OXPHOS) to aerobic glycolysis to generate lactate and ATP even in the presence of $\mathrm{O}_{2}$ (i.e., Warburg effect) [2]. Since the Warburg effect is also found in tumor cells with intact and functional mitochondria, it is reasonable to assume that it could represent a strategy adopted by cancer cells, not only to cope with the greater energy demands but also to reduce oxidative stress, preserving cells from oxidative death [3]. In this regard, reactive oxygen species (ROS), maintained at "physiological" levels, have been demonstrated to activate redox signaling pathways involved in cell proliferation and survival $[4,5]$.
Over the past decade, numerous studies have supported the hypothesis that the Warburg effect can be explained by the alterations in multiple signaling pathways resulting from mutations of oncogenes and tumor suppressor genes $[6,7]$. Indeed, tumor metabolic reprogramming involves the activation of key metabolic pathways such as glycolysis, the pentose phosphate pathway, and glutaminolysis [8].

In this regard, it has been demonstrated that the glycolytic metabolic switch is due to a marked slowing down of the conversion of phosphoenolpyruvate into pyruvate, a reaction catalyzed by pyruvate kinase (PKM) [9]. Furthermore, in cancer cells, it has been observed that the presence of the low-activity dimeric form of PKM2 promotes the conversion of pyruvate to lactate [10] and that the increased levels of lactic acid detected in cancer patients are related to rapid tumor growth and high levels of metastases [11]. Moreover, considering that most chemotherapeutic agents are weak bases, the presence of lactic acid, generating acidity, induces the ionization of the drugs which, in their modified 
chemical structure, are not able to enter the tumor cells, thus facilitating the onset of chemoresistance $[12,13]$.

PKM2, which makes cells less susceptible to oxidative stress and enhances NADPH production [14, 15], has been found to have a role in chemoresistance. In fact, a recent study showed that this kinase promotes gemcitabine resistance on one hand by inhibiting the transcriptional activation of p53 and the p38-mediated signaling pathway and on the other by increasing the expression of the antiapoptotic protein bcl-xl [16]. In addition, it has been reported that many cancer cells in order to satisfy their bioenergetic and metabolic needs depend on glutamine which is the main source of tricarboxylic acid (TCA) cycle precursors (Figure 1). For example, at the mitochondrial level, glutamine is converted to glutamate by glutaminase (GLS). In turn, glutamate can be converted to $\alpha$-ketoglutarate (KG) by glutamate dehydrogenase $(\mathrm{GDH})$ or transaminase, resulting in sustaining the TCA cycle. In addition, glutamate can serve as a precursor not only of nonessential amino acids such as aspartate, alanine, proline, and arginine but also of the most important intracellular antioxidant, glutathione $(\mathrm{GSH})$, which is a tripeptide consisting of glutamate, cysteine, and glycine. In addition, malate, which is derived from glutamine, can be converted into pyruvate, leading to NADPH formation [17]. Therefore, the production of NADPH and GSH, derived from glutamine, allows cancer cells to reduce oxidative stress levels associated with mitochondrial respiration and rapid cell proliferation (Figure 1).

In this regard, our recent studies on human neuroblastoma (NB) cells [18], as well as other studies carried out on brain tumor samples [19] and ovarian cancer cells [20], have all demonstrated that the acquisition of chemoresistance is associated with high levels of GSH that enable cancer cells to counteract the prooxidant action of many chemotherapeutic agents $[4,21,22]$.

It is noteworthy that the dependency of tumors on specific metabolic substrates, such as glucose or glutamine, is determined by alterations in oncogenes and oncosuppressor genes which are responsible for the tumor metabolic phenotype, while also supporting tumorigenesis. Among oncogenes, MYC has been found to have a pivotal role in the metabolic reprogramming of tumor cells by enhancing glucose uptake and glycolysis, lactate production and export, glutamine uptake and glutaminolysis, mitochondrial biogenesis, and oxidative phosphorylation [1].

\section{Role of MYC in Cancer Metabolic Reprogramming and Adaptation to Therapy}

MYC is a family of protooncogenes (i.e., c-MYC, L-MYC and N-MYC) which encode transcription factors that have roles in both normal and cancer cell physiologies. MYC requires dimerization with the protein MAX for DNA binding and for the assembly of transcriptional machinery. MAX can also interact with Mxd members which are transcriptional repressors and act in antagonism with MYC/MAX complexes. In addition, Mxd members can also bind to Mlx proteins that can interact with transcription activators of the Mondo family [23]. The MondoA/Mlx complex, located in the cytosol, translocates to the nucleus where, in response to an increase in extracellular glucose levels, it stimulates the expression of the thioredoxin-interacting protein (TXNIP) which suppresses the glucose uptake by limiting the expression of glucose transporters (GLUT) in the membrane [24, 25].

MYC is strongly involved in regulating cell metabolism and facilitates glycolysis by inducing the activation of genes encoding for glycolytic enzymes and GLUT (Figure 1) [26]. It is also able to promote mitochondrial biogenesis and function, thus increasing both oxygen consumption and ATP production [27-29].

Furthermore, it has been found that MYC upregulates the expression of glutamine transporters, facilitating glutaminolysis $[30,31]$, which is also stimulated by repressing microRNA-23a/b transcription leading to GLS1 overexpression [32]. As reported above, GLS converts glutamine to glutamate [32] which either enters the TCA cycle for the production of ATP or serves as a substrate for GSH synthesis [30]. In this regard, it has been reported that $\mathrm{S} 6 \mathrm{~K} 1$, a downstream effector of mTORC1, facilitates the translation of MYC, further contributing to the increase of GLS and GDH $[33,34]$. In addition, it has been shown that mTORC1 expression, in response to stress conditions, is inhibited by FOXO transcription factors [35] and an increased expression of FOXO3a is able to antagonize the MYC binding to promoters, reducing the mitochondrial mass, oxygen consumption, and ROS production [36].

Regarding the enhancement of the mitochondrial function, it has been found that MYC can activate the PPAR $\gamma$ coactivator- $1 \alpha$ (PGC- $1 \alpha$ ) and the mitochondrial transcription factor A (TFAM), mediators of mitochondrial biogenesis and mitochondrial gene expression, respectively [28, 37]. Interestingly, although the role played by MYC/PGC- $1 \alpha$ axis is controversial [38], several reports have demonstrated that PGC1- $\alpha$ is involved in chemoresistance [39] and the inhibition of the PGC- $1 \alpha$ pathway has been found to activate glycolysis [40] and to sensitize melanoma to oxidative damage [41].

Therefore, as reported above, the MYC-overexpressing tumors depend on glutamine $[30,31]$, and it has been demonstrated that glutamine depletion leads to the reduction of GSH levels and consequently triggers apoptosis. In fact, buthionine sulfoximine- (BSO-) induced depletion of GSH was able to induce apoptosis of N-MYC-amplified NB cells through a ROS-mediated activation of $\mathrm{PKC} \delta$-dependent pathways (Figure 2) [5, 42, 43]. Accordingly, PKC $\delta$ overexpression sensitized NB cells to the proapoptotic effects of BSO and of etoposide [18, 44-46].

Clinical studies carried out on NB patients have demonstrated that N-MYC amplification correlates to a reduction in the survival rate of those patients undergoing a multidrug therapy protocol consisting of etoposide, vincristine, carboplatin, adriamycin, and cyclophosphamide [47].

\section{Molecular Mechanisms of MYC-Dependent Metabolic Changes}

In N-MYC-amplified NB tumors, Akt has been found to be hyperactivated [48] and Akt activation has been demonstrated 


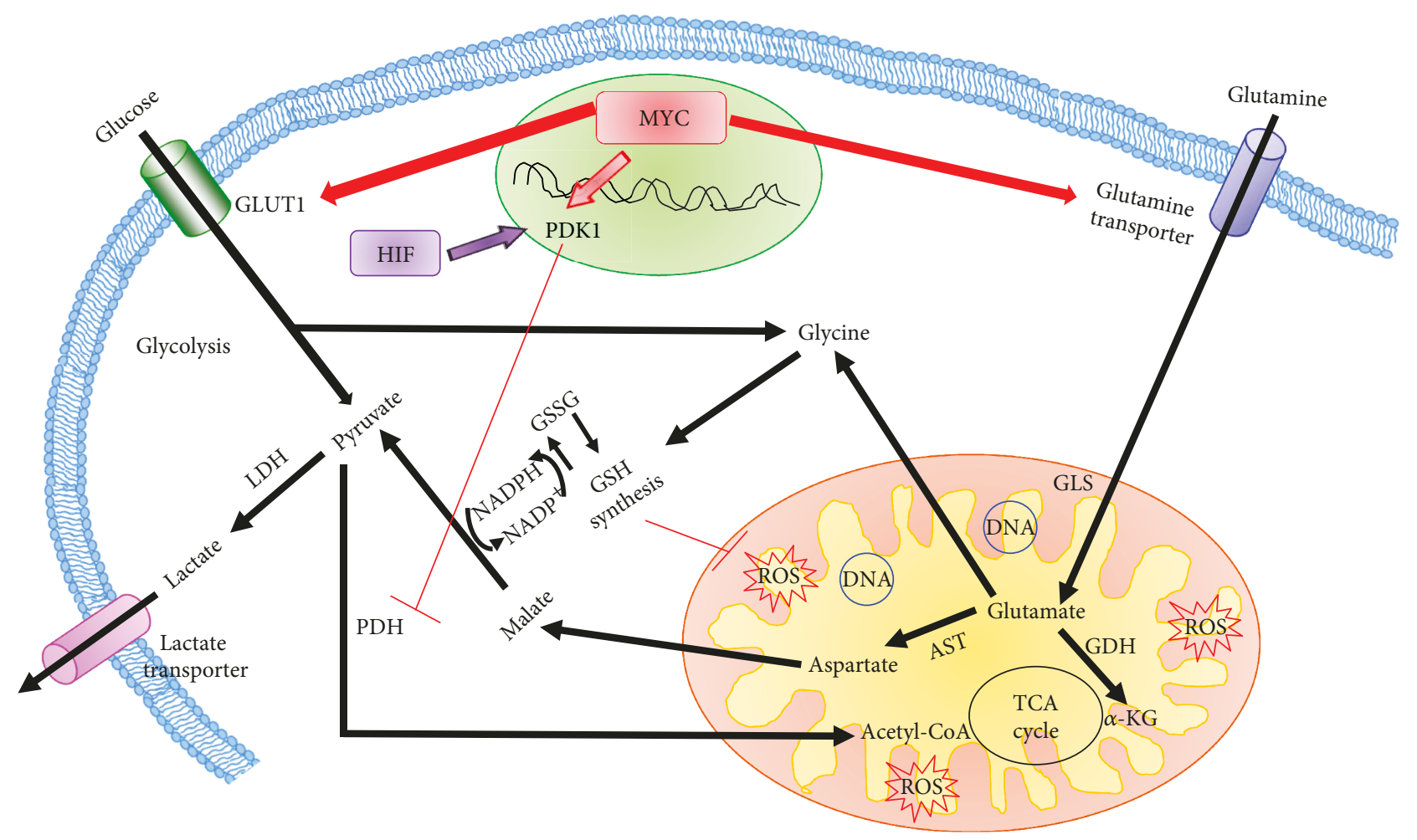

Figure 1: Role of MYC in the modulation of cancer metabolic reprogramming. MYC is involved in the modulation of both glycolysis and glutaminolysis. MYC, in order to carry out this double role, upregulates membrane transporters and enzymes involved in these metabolic processes (indicated in red). AST: glutamic-oxaloacetic transaminase; GDH: glutamate dehydrogenase; GLS: glutaminase; GLUT1: glucose transporter 1; GSH: reduced glutathione; GSSG: oxidized glutathione; $\alpha$-KG: $\alpha$-ketoglutarate; LDH: lactic dehydrogenase; PDH: pyruvate dehydrogenase; PDK1: pyruvate dehydrogenase kinase 1; ROS: reactive oxygen species; TCA: tricarboxylic acid.

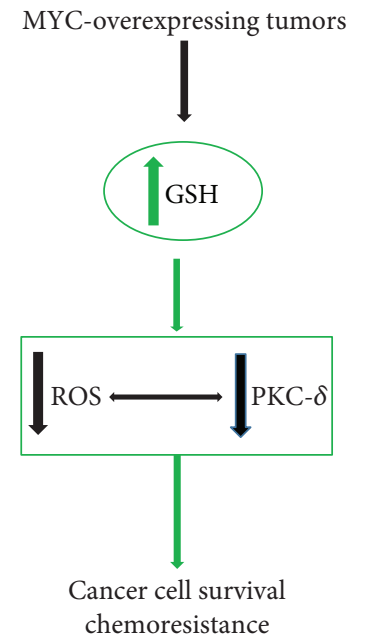

(a)

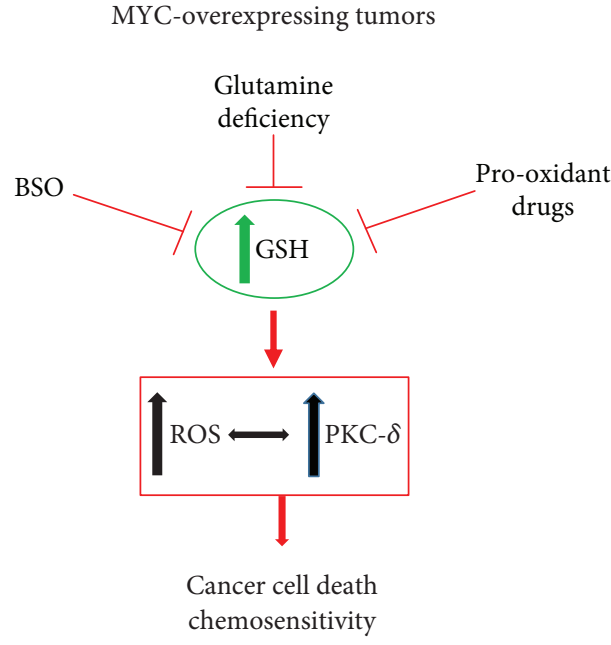

(b)

FIGURE 2: MYC overexpression and increase of glutathione levels in the acquisition of chemoresistance. (a) Chemoresistance of MYCoverexpressing tumors is associated with an enhancement of intracellular glutathione (GSH) levels. (b) In order to promote cell death, it is helpful to deplete GSH by using depleting agents such as buthionine sulfoximine (BSO) or prooxidant drugs. These strategies stimulate reactive oxygen species (ROS) production which modulate, and are modulated by, the proapoptotic protein kinase C-delta (PKC- $\delta$ ).

to be strongly involved in etoposide resistance [46, 49-51], as well as being related to the expression of CD133, a marker of staminality associated with the most aggressive cancer phenotype [52]. Accordingly, it has been shown that
Akt inhibition sensitizes NB cells to the cytotoxic action of etoposide [53], doxorubicin, vincristine, and cisplatin [52]. In addition, under conditions of nutrient deficiency, the reduced activity of Akt decreases the amount of MDM2, 
the p53 endogenous inhibitor, resulting in an increase in p53 levels [54]. In fact, it has been found that the activation of p53 limits glycolysis and promotes OXPHOS in cancer cells while the loss of function of mutated p53 contributes to the development of the Warburg effect $[55,56]$. Therefore, $\mathrm{p} 53$, in repressing PGC- $1 \alpha$, which is involved in mitochondrial biogenesis, and modulating other genes implicated in autophagy, in glucose metabolism, and also in the pentosephosphate pathway [57-66], can play a role as a regulator of tumor cell metabolism and chemoresistance.

Interestingly, our recent studies have shown that chronic treatment of N-MYC-amplified NB cells with etoposide does not modify the homozygous p53 mutation (A161T), previously found in etoposide-sensitive NB cells, and therefore, in this context, p53 is responsible neither for OXPHOS activation nor for the metabolic adaptation of etoposideresistant NB cells [67].

Moreover, several studies have demonstrated that the metabolic reprogramming might be the result of the "molecular interplay" between N-MYC and hypoxia-inducible factors (HIFs) [68]. HIF1 and HIF2 provide transcriptional homeostatic responses to limited oxygen levels in both physiological and pathological conditions. Although physiological HIF1 can inhibit the activity of normal MYC, the altered expression of the oncogenic MYC collaborates with HIF to confer the propensity to cancer cells to convert glucose to lactate, even in the presence of adequate $\mathrm{O}_{2}$ levels [69-72]. In fact, at normal MYC levels, it has been observed that HIF $1 \alpha$ can compete for MAX, displacing MYC, while, at higher MYC levels, the formation of MYC-MAX heterodimers is maintained through mass action. Similar to MYC, HIF1 activates all genes involved in glycolysis, but unlike MYC, HIF1 actively inhibits mitochondrial respiration by promoting mitochondrial autophagy $[73,74]$ and preventing mitochondrial biogenesis [29]. In this context, it has been reported that HIF1 induces the expression of pyruvate dehydrogenase kinase (PDK1) which phosphorylates and inactivates pyruvate dehydrogenase, a mitochondrial enzyme catalyzing the conversion of pyruvate to acetyl CoA [75, 76]. Moreover, it has been found that MYC, when overexpressed in human tumors, cooperates with HIF1 to induce PDK1 and hexokinase 2 (HK2) expression, altering cellular metabolism in favor of glycolysis with an increased production of lactate [70, 75]. HIF1 and MYC independently activate GLUT1 and lactate dehydrogenase A (LDHA), resulting in an increased glucose influx and higher glycolytic rates [75].

Interestingly, HK2, which plays a key role for the Warburg effect in cancer, binds competitively to the voltage-dependent anion channel (VDAC), in the outer mitochondrial membrane, preventing its union with proapoptotic Bax and thereby avoiding apoptosis [77].

Apoptosis and senescence represent two tumorsuppressive mechanisms which can be modulated by MYC and RAS oncogenes. In fact, RAS inhibits MYC-induced apoptosis via PI3K activity and MYC suppresses RAS-induced senescence via CdK2, a cyclin-dependent kinase which phosphorylates MYC at Ser62 residue [78]. Accordingly, CdK2 inhibition has been shown to slow down the growth of
MYCN-amplified neuroblastoma cells [79] and of other MYC-driven tumors [80].

Many chemotherapeutic drugs exert their cytotoxic effects on cancer cells by reactivating apoptosis and/or senescence [81]. In this context, it has been hypothesized that therapy-induced senescence (TIS) could be useful in the treatment of tumors with an impairment of the apoptotic pathways.

Interestingly, it is relevant to know that the presence of TIS cells can stimulate immunosurveillance and also induce chemoresistance $[82,83]$. In fact, TIS cells have features of stemness that is regulated by the Wnt-dependent pathways [84-86] and undergo a metabolic reprogramming characterized by an increase in the glycolytic activity $[2,3]$ and an impairment of proteasome activity and autophagy [87]. In this context, the treatment of oncologic patients with anthracyclines and alkylating agents has been shown to induce cellular senescence and the secretion of cytokines, chemokines, growth factors, and proteases that can contribute to the side effects of chemotherapy $[82,88]$.

Recently, it has been reported that downregulation of p21, a cell cycle inhibitor, leads to MYC upregulation which represses the expression of $\mathrm{CD} 47$ receptor generating a subpopulation of cells that escape senescence [89]. However, further studies are necessary to determine if senescence is a general adaptive pathway to chemotherapy and if this response concerns only a specific subpopulation of cancer cells.

\section{Inhibition of MYC Effectors as a Potential Strategy to Block Cancer Metabolic Reprogramming}

Although MYC is considered the "most-wanted" target for anticancer therapy [90], the targeting of this oncogene has not yet obtained any positive outcomes. In fact, the inhibition of MYC can interfere with its physiological functions and therefore an alternative approach inhibiting MYC effectors could be more useful. More specifically, given that MYC drives the glucose and glutamine metabolism of cancer cells, the use of small molecules, able to inhibit enzymes involved in glycolysis and glutaminolysis, might be effective in slowing down tumor cell proliferation. Among them, several drugs targeting the MYC effectors are currently being tested in clinical practice [91-97] (Table 1).

Interestingly, a promising approach could be to indirectly modulate MYC through the "synthetic lethality" [90], and in this regard, the development of MK-3475 (pembrolizumab or keytruda) might offer new therapeutic opportunities. In fact, this latter compound is an inhibitor of the programmed death-1 (PD-1) protein and MYC modulates the expression of its ligand (PD-L1) [98], which, when overexpressed, stimulates glucose metabolism [99] by increasing GLUT1 expression [100]. MK-3475 has been, and is currently, the subject of over 900 clinical trials, and two of these have even reached Phase 4 (NCT03715205; NCT03134456). In addition, in Phase 3 studies, it should be noted that this compound per se is efficacious in treating recurrent or metastatic head- 
TABLe 1: Drugs targeting glucose or glutamine metabolism currently used in clinical trials.

\begin{tabular}{|c|c|c|c|c|c|}
\hline Drug & Target & Effect on MYC & Cancer type & Phase trials & NCT \\
\hline Silibyn & GLUT & Reduction [91] & Prostate cancer & II & 00487721 \\
\hline \multirow{5}{*}{ Gossypol } & \multirow{5}{*}{ Lactate dehydrogenase (LDH) } & \multirow{5}{*}{ Reduction [92] } & Small-cell lung carcinoma & II & 00773955 \\
\hline & & & Prostate cancer & II & 00666666 \\
\hline & & & Esophageal/gastroesophageal cancer & $\mathrm{I} / \mathrm{II}$ & 00561197 \\
\hline & & & \multirow{2}{*}{ Glioblastoma } & I & 00390403 \\
\hline & & & & II & 00540722 \\
\hline \multirow{3}{*}{ Dichloroacetate } & \multirow{3}{*}{ Pyruvate dehydrogenase (PDH) } & \multirow{3}{*}{ Reduction [93] } & $\begin{array}{c}\text { Breast cancer and non-small-cell } \\
\text { lung carcinoma }\end{array}$ & II & 01029925 \\
\hline & & & Head and neck cancer & I & 01163487 \\
\hline & & & Brain cancer & II & 00540176 \\
\hline \multirow{2}{*}{ Deoxyglucose } & \multirow{2}{*}{ Hexokinase II } & \multirow{2}{*}{ Reduction [94] } & Prostate cancer & $\mathrm{I} / \mathrm{II}$ & 00633087 \\
\hline & & & Lung cancer and breast cancer & I & 00096707 \\
\hline Apigenin & Pyruvate kinase M (PKM) & Reduction [95] & Breast cancer & - & 03139227 \\
\hline Diclofenac & GLUT1 and LDH & Reduction [96] & Basal cell carcinoma & II & 01358045 \\
\hline \multirow{5}{*}{ CB-839 } & \multirow{5}{*}{ Glutaminase1 (GLS1) } & \multirow{5}{*}{ Reduction [97] } & Leukemia & I & 02071927 \\
\hline & & & Colorectal cancer & $\mathrm{I} / \mathrm{II}$ & 02861300 \\
\hline & & & Hematological tumors & I & 02071888 \\
\hline & & & Melanoma & $\mathrm{I} / \mathrm{II}$ & 02771626 \\
\hline & & & $\begin{array}{c}\text { Triple negative breast cancer and } \\
\text { solid tumors }\end{array}$ & I & 02071862 \\
\hline
\end{tabular}

and-neck squamous cell carcinoma (NCT02252042) [101], advanced urothelial carcinoma (NCT02256436) [102], nonsmall-cell lung cancer (NCT01905657) [103], and melanoma (NCT02362594) [104].

\section{Conclusions}

Tumor metabolic reprogramming is a direct result of the reengineering of intracellular signaling pathways that are altered by activated oncogenes or downregulated oncosuppressors and by epigenetic changes, conferring a proliferative advantage to cancer cells.

Indeed, tumors may prefer either a glycolytic or an oxidative metabolism, depending on the activation of oncogenes or repression of oncosuppressors but also on the tumor microenvironment. Therefore, it is conceivable that in the tumor niche there is a strong "metabolic competition" due to high nutritional requirements and also an intense "molecular interplay" able to maintain an efficient metabolism. The balance between these factors could paradoxically guarantee the development and the survival of cancer even under therapyinduced stress conditions. Consequently, therapies that block glucose metabolism might be more effective towards tumors with high glycolytic rates, while they might develop therapy resistance in tumors whose metabolism depends on oxidative phosphorylation [105].

Therefore, anticancer therapy must take into account that most chemotherapeutic drugs are prooxidant agents and are able to induce a metabolic reprogramming that alters the redox homeostasis of cancer cells activating signaling pathways responsible for cell survival.
Considering the crucial role of MYC in driving the metabolic reprogramming of cancer which has been shown to be strictly related to drug resistance, several studies have been carried out in order to focus MYC-dependent metabolic pathways. Even though the efforts are multiple, to date the applicability of MYC inhibitors is still a utopia. However, the use of small molecules, able to inhibit MYC-related enzymes involved in glycolysis and glutaminolysis, might result effective in slowing down tumor cell proliferation and counteracting chemoresistance.

However, the characterization of the metabolic reprogramming of tumors and its connection with oncogenic signaling is a promising strategy to identify novel molecular approaches in anticancer treatment.

\section{Conflicts of Interest}

The authors declare that there is no conflict of interest regarding the publication of this paper.

\section{Acknowledgments}

This work was supported by grants from Genoa University. We would like to thank Mr. Giuseppe Catalano (DIMES, University of Genoa) for his technical assistance and Ms. Suzanne Patten for the language editing.

\section{References}

[1] M. Tarrado-Castellarnau, P. de Atauri, and M. Cascante, "Oncogenic regulation of tumor metabolic reprogramming," Oncotarget, vol. 7, no. 38, pp. 62726-62753, 2016. 
[2] O. Warburg, F. Wind, and N. Negelein, "The metabolism of tumors in the body," Journal of General Physiology, vol. 8, no. 6, pp. 519-530, 1927.

[3] M. G. Vander Heiden, L. C. Cantley, and C. B. Thompson, "Understanding the Warburg effect: the metabolic requirements of cell proliferation," Science, vol. 324, no. 5930, pp. 1029-1033, 2009.

[4] B. Marengo, M. Nitti, A. L. Furfaro et al., "Redox homeostasis and cellular antioxidant systems: crucial players in cancer growth and therapy," Oxidative Medicine and Cellular Longevity, vol. 2016, Article ID 6235641, 16 pages, 2016.

[5] B. Marengo, L. Raffaghello, V. Pistoia et al., "Reactive oxygen species: biological stimuli of neuroblastoma cell response," Cancer Letters, vol. 228, no. 1-2, pp. 111-116, 2005.

[6] J. P. Bayley and P. Devilee, "The Warburg effect in 2012," Current Opinion in Oncology, vol. 24, no. 1, pp. 62-67, 2012.

[7] M. V. Liberti and J. W. Locasale, "The Warburg effect: how does it benefit cancer cells?," Trends in Biochemical Sciences, vol. 41, no. 3, pp. 211-218, 2016.

[8] P. S. Ward and C. B. Thompson, "Metabolic reprogramming: a cancer hallmark even Warburg did not anticipate," Cancer Cell, vol. 21, no. 3, pp. 297-308, 2012.

[9] H. R. Christofk, M. G. Vander Heiden, M. H. Harris et al., "The M2 splice isoform of pyruvate kinase is important for cancer metabolism and tumour growth," Nature, vol. 452, no. 7184, pp. 230-233, 2008.

[10] M. Tamada, M. Suematsu, and H. Saya, "Pyruvate kinase M2: multiple faces for conferring benefits on cancer cells," Clinical Cancer Research, vol. 18, no. 20, pp. 5554-5561, 2012.

[11] S. Walenta and W. F. Mueller-Klieser, "Lactate: mirror and motor of tumor malignancy," Seminars in Radiation Oncology, vol. 14, no. 3, pp. 267-274, 2004.

[12] R. Amorim, C. Pinheiro, V. Miranda-Gonçalves et al., "Monocarboxylate transport inhibition potentiates the cytotoxic effect of 5-fluorouracil in colorectal cancer cells," Cancer Letters, vol. 365, no. 1, pp. 68-78, 2015.

[13] N. Draoui, O. Schicke, E. Seront et al., "Antitumor activity of 7-aminocarboxycoumarin derivatives, a new class of potent inhibitors of lactate influx but not efflux," Molecular Cancer Therapeutics, vol. 13, no. 6, pp. 1410-1418, 2014.

[14] D. Anastasiou, G. Poulogiannis, J. M. Asara et al., "Inhibition of pyruvate kinase M2 by reactive oxygen species contributes to cellular antioxidant responses," Science, vol. 334, no. 6060, pp. 1278-1283, 2011.

[15] R. B. Hamanaka and N. S. Chandel, "Warburg effect and redox balance," Science, vol. 334, no. 6060, pp. 1219-1220, 2011.

[16] D. J. Kim, Y. S. Park, M. G. Kang et al., "Pyruvate kinase isoenzyme M2 is a therapeutic target of gemcitabine-resistant pancreatic cancer cells," Experimental Cell Research, vol. 336, no. 1, pp. 119-129, 2015.

[17] R. J. DeBerardinis, A. Mancuso, E. Daikhin et al., "Beyond aerobic glycolysis: transformed cells can engage in glutamine metabolism that exceeds the requirement for protein and nucleotide synthesis," Proceedings of the National Academy of Sciences of the United States of America, vol. 104, no. 49, pp. 19345-19350, 2007.

[18] R. Colla, A. Izzotti, C. de Ciucis et al., "Glutathione-mediated antioxidant response and aerobic metabolism: two crucial factors involved in determining the multi-drug resistance of high-risk neuroblastoma," Oncotarget, vol. 7, no. 43, pp. 70715-70737, 2016.

[19] D. S. Backos, C. C. Franklin, and P. Reigan, "The role of glutathione in brain tumor drug resistance," Biochemical Pharmacology, vol. 83, no. 8, pp. 1005-1012, 2012.

[20] E. Wang, S. Bhattacharyya, A. Szabolcs et al., "Enhancing chemotherapy response with Bmi-1 silencing in ovarian cancer," PLoS One, vol. 6, no. 3, article e17918, 2011.

[21] N. Traverso, R. Ricciarelli, M. Nitti et al., "Role of glutathione in cancer progression and chemoresistance," Oxidative Medicine and Cellular Longevity, vol. 2013, Article ID 972913, 10 pages, 2013.

[22] J. Cen, L. Zhang, F. Liu, F. Zhang, and B. S. Ji, "Long-term alteration of reactive oxygen species led to multidrug resistance in MCF-7 cells," Oxidative Medicine and Cellular Longevity, vol. 2016, Article ID 7053451, 15 pages, 2016.

[23] B. M. Iritani, J. Delrow, C. Grandori et al., "Modulation of T-lymphocyte development, growth and cell size by the Myc antagonist and transcriptional repressor Mad1," The EMBO Journal, vol. 21, no. 18, pp. 4820-4830, 2002.

[24] B. R. Wilde and D. E. Ayer, "Interactions between Myc and MondoA transcription factors in metabolism and tumourigenesis," British Journal of Cancer, vol. 113, no. 11, pp. 1529-1533, 2015.

[25] N. Wu, B. Zheng, A. Shaywitz et al., "AMPK-dependent degradation of TXNIP upon energy stress leads to enhanced glucose uptake via GLUT1," Molecular Cell, vol. 49, no. 6, pp. 1167-1175, 2013.

[26] R. C. Osthus, H. Shim, S. Kim et al., "Deregulation of glucose transporter 1 and glycolytic gene expression by c-Myc," Journal of Biological Chemistry, vol. 275, no. 29, pp. 21797-21800, 2000 .

[27] T. Wahlström and M. Arsenian Henriksson, "Impact of MYC in regulation of tumor cell metabolism," Biochimica et Biophysica Acta (BBA) - Gene Regulatory Mechanisms, vol. 1849, no. 5, pp. 563-569, 2015.

[28] F. Li, Y. Wang, K. I. Zeller et al., "Myc stimulates nuclearly encoded mitochondrial genes and mitochondrial biogenesis," Molecular and Cellular Biology, vol. 25, no. 14, pp. 62256234, 2005.

[29] H. Zhang, P. Gao, R. Fukuda et al., "HIF-1 inhibits mitochondrial biogenesis and cellular respiration in VHL-deficient renal cell carcinoma by repression of C-MYC activity," Cancer Cell, vol. 11, no. 5, pp. 407-420, 2007.

[30] D. R. Wise, R. J. DeBerardinis, A. Mancuso et al., "Myc regulates a transcriptional program that stimulates mitochondrial glutaminolysis and leads to glutamine addiction," Proceedings of the National Academy of Sciences of the United States of America, vol. 105, no. 48, pp. 18782-18787, 2008.

[31] M. O. Yuneva, T. W. M. Fan, T. D. Allen et al., "The metabolic profile of tumors depends on both the responsible genetic lesion and tissue type," Cell Metabolism, vol. 15, no. 2, pp. 157-170, 2012.

[32] P. Gao, I. Tchernyshyov, T.-C. Chang et al., "C-Myc suppression of miR-23a/b enhances mitochondrial glutaminase expression and glutamine metabolism," Nature, vol. 458, no. 7239, pp. 762-765, 2009.

[33] A. Csibi, G. Lee, S.-O. Yoon et al., "The mTORC1/S6K1 pathway regulates glutamine metabolism through the eIF4Bdependent control of c-Myc translation," Current Biology, vol. 24, no. 19, pp. 2274-2280, 2014. 
[34] A. Csibi, S. M. Fendt, C. Li et al., "The mTORC1 pathway stimulates glutamine metabolism and cell proliferation by repressing SIRT4," Cell, vol. 153, no. 4, pp. 840-854, 2013.

[35] A. Lin, J. Yao, L. Zhuang et al., "The FoxO-BNIP3 axis exerts a unique regulation of mTORC1 and cell survival under energy stress," Oncogene, vol. 33, no. 24, pp. 3183-3194, 2014.

[36] K. S. Jensen, T. Binderup, K. T. Jensen et al., "FoxO3A promotes metabolic adaptation to hypoxia by antagonizing Myc function," The EMBO Journal, vol. 30, no. 22, pp. 4554-4570, 2011.

[37] D. P. Kelly and R. C. Scarpulla, "Transcriptional regulatory circuits controlling mitochondrial biogenesis and function," Genes \& Development, vol. 18, no. 4, pp. 357-368, 2004.

[38] P. Sancho, E. Burgos-Ramos, A. Tavera et al., "MYC/PGC- $1 \alpha$ balance determines the metabolic phenotype and plasticity of pancreatic cancer stem cells," Cell Metabolism, vol. 22, no. 4, pp. 590-605, 2015.

[39] V. S. LeBleu, J. T. O’Connell, K. N. Gonzalez Herrera et al., "PGC- $1 \alpha$ mediates mitochondrial biogenesis and oxidative phosphorylation in cancer cells to promote metastasis," Nature Cell Biology, vol. 16, no. 10, pp. 992-1003, 2014.

[40] J. H. Lim, C. Luo, F. Vazquez, and P. Puigserver, "Targeting mitochondrial oxidative metabolism in melanoma causes metabolic compensation through glucose and glutamine utilization," Cancer Research, vol. 74, no. 13, pp. 3535-3545, 2014.

[41] F. Vazquez, J. H. Lim, H. Chim et al., "PGC1 $\alpha$ expression defines a subset of human melanoma tumors with increased mitochondrial capacity and resistance to oxidative stress," Cancer Cell, vol. 23, no. 3, pp. 287-301, 2013.

[42] B. Marengo, C. de Ciucis, D. Verzola et al., "Mechanisms of BSO (L-buthionine-S,R-sulfoximine)-induced cytotoxic effects in neuroblastoma," Free Radical Biology \& Medicine, vol. 44, no. 3, pp. 474-482, 2008.

[43] B. Marengo, C. De Ciucis, R. Ricciarelli, M. A. Pronzato, U. M. Marinari, and C. Domenicotti, "Protein kinase C: an attractive target for cancer therapy," Cancers, vol. 3, no. 1, pp. 531-567, 2011.

[44] B. Marengo, C. de Ciucis, R. Ricciarelli et al., "PKC $\delta$ sensitizes neuroblastoma cells to L-buthionine-sulfoximine and etoposide inducing reactive oxygen species overproduction and DNA damage," PLoS One, vol. 6, no. 2, article e14661, 2011.

[45] C. Domenicotti, B. Marengo, M. Nitti et al., "A novel role of protein kinase C-delta in cell signaling triggered by glutathione depletion," Biochemical Pharmacology, vol. 66, no. 8, pp. 1521-1526, 2003.

[46] B. Marengo, C. G. de Ciucis, R. Ricciarelli et al., "p38MAPK inhibition: a new combined approach to reduce neuroblastoma resistance under etoposide treatment," Cell Death \& Disease, vol. 4, no. 4, article e589, 2013.

[47] Z. Y. Zhong, B. J. Shi, H. Zhou, and W. B. Wang, "CD133 expression and MYCN amplification induce chemoresistance and reduce average survival time in pediatric neuroblastoma," Journal of International Medical Research, vol. 46, no. 3, pp. 1209-1220, 2018.

[48] A. Nakagawara, M. Arima-Nakagawara, N. J. Scavarda, C. G. Azar, A. B. Cantor, and G. M. Brodeur, "Association between high levels of expression of the TRK gene and favorable outcome in human neuroblastoma," The New England Journal of Medicine, vol. 328, no. 12, pp. 847-854, 1993.
[49] S. Scala, K. Wosikowski, P. Giannakakou et al., "Brainderived neurotrophic factor protects neuroblastoma cells from vinblastine toxicity," Cancer Research, vol. 56, no. 16, pp. 3737-3742, 1996.

[50] J. Jaboin, C. J. Kim, D. R. Kaplan, and C. J. Thiele, "Brainderived neurotrophic factor activation of TrkB protects neuroblastoma cells from chemotherapy-induced apoptosis via phosphatidylinositol 3'-kinase pathway," Cancer Research, vol. 62, no. 22, pp. 6756-6763, 2002.

[51] Z. Li, J. Jaboin, P. A. Dennis, and C. J. Thiele, "Genetic and pharmacologic identification of Akt as a mediator of brain-derived neurotrophic factor/TrkB rescue of neuroblastoma cells from chemotherapy-induced cell death," Cancer Research, vol. 65, no. 6, pp. 2070-2075, 2005.

[52] H. Sartelet, T. Imbriglio, C. Nyalendo et al., "CD133 expression is associated with poor outcome in neuroblastoma via chemoresistance mediated by the AKT pathway," Histopathology, vol. 60, no. 7, pp. 1144-1155, 2012.

[53] Z. Li, D. Y. Oh, K. Nakamura, and C. J. Thiele, "Perifosine-induced inhibition of Akt attenuates brain-derived neurotrophic factor/TrkB-induced chemoresistance in neuroblastoma in vivo," Cancer, vol. 117, no. 23, pp. 54125422, 2011.

[54] B. P. Zhou, Y. Liao, W. Xia, Y. Zou, B. Spohn, and M. C. Hung, "HER-2/neu induces p53 ubiquitination via Aktmediated MDM2 phosphorylation," Nature Cell Biology, vol. 3, no. 11, pp. 973-982, 2001.

[55] R. A. Cairns, I. S. Harris, and T. W. Mak, "Regulation of cancer cell metabolism," Nature Reviews Cancer, vol. 11, no. 2, pp. 85-95, 2011.

[56] A. J. Levine and A. M. Puzio-Kuter, "The control of the metabolic switch in cancers by oncogenes and tumor suppressor genes," Science, vol. 330, no. 6009, pp. 1340-1344, 2010.

[57] D. Crighton, S. Wilkinson, J. O'Prey et al., "DRAM, a p53induced modulator of autophagy, is critical for apoptosis," Cell, vol. 126, no. 1, pp. 121-134, 2006.

[58] D. Kenzelmann Broz, S. Spano Mello, K. T. Bieging et al., "Global genomic profiling reveals an extensive p53-regulated autophagy program contributing to key p53 responses," Genes \& Development, vol. 27, no. 9, pp. 1016-1031, 2013.

[59] K. H. Vousden and X. Lu, "Live or let die: the cell's response to p53," Nature Reviews Cancer, vol. 2, no. 8, pp. 594-604, 2002.

[60] K. H. Vousden and K. M. Ryan, "p53 and metabolism," Nature Reviews Cancer, vol. 9, no. 10, pp. 691-700, 2009.

[61] E. White, "Autophagy and p53," Cold Spring Harbor Perspectives in Medicine, vol. 6, no. 4, article a026120, 2016.

[62] K. Sajnani, F. Islam, R. A. Smith, V. Gopalan, and A. K. Y. Lam, "Genetic alterations in Krebs cycle and its impact on cancer pathogenesis," Biochimie, vol. 135, pp. 164-172, 2017.

[63] W. X. Mai, L. Gosa, V. W. Daniels et al., "Cytoplasmic p53 couples oncogene-driven glucose metabolism to apoptosis and is a therapeutic target in glioblastoma," Nature Medicine, vol. 23, no. 11, pp. 1342-1351, 2017.

[64] F. M. Simabuco, M. G. Morale, I. C. B. Pavan, A. P. Morelli, F. R. Silva, and R. E. Tamura, "p53 and metabolism: from mechanism to therapeutics," Oncotarget, vol. 9, no. 34, pp. 23780-23823, 2018.

[65] M. C. De Santis, P. E. Porporato, M. Martini, and A. Morandi, "Signaling pathways regulating redox balance in cancer metabolism," Frontiers in Oncology, vol. 8, no. 126, 2018. 
[66] I. Hernández-Reséndiz, J. C. Gallardo-Pérez, A. López-Macay et al., "Mutant p53 $3^{\mathrm{R} 248 \mathrm{Q}}$ downregulates oxidative phosphorylation and upregulates glycolysis under normoxia and hypoxia in human cervix cancer cells," Journal of Cellular Physiology, vol. 234, no. 5, pp. 5524-5536, 2018.

[67] B. Marengo, P. Monti, M. Miele et al., "Etoposide-resistance in a neuroblastoma model cell line is associated with 13q14.3 mono-allelic deletion and miRNA-15a/16-1 downregulation," Scientific Reports, vol. 8, no. 1, article 13762 , 2018.

[68] G. Qing, N. Skuli, P. A. Mayes et al., “Combinatorial regulation of neuroblastoma tumor progression by $\mathrm{N}-\mathrm{Myc}$ and hypoxia inducible factor HIF-1 $\alpha$," Cancer Research, vol. 70, no. 24, pp. 10351-10361, 2010.

[69] M. Koshiji, Y. Kageyama, E. A. Pete, I. Horikawa, J. C. Barrett, and L. E. Huang, "HIF- $1 \alpha$ induces cell cycle arrest by functionally counteracting Myc," The EMBO Journal, vol. 23, no. 9, pp. 1949-1956, 2004.

[70] J. D. Gordan, C. B. Thompson, and M. C. Simon, "HIF and cMyc: sibling rivals for control of cancer cell metabolism and proliferation," Cancer Cell, vol. 12, no. 2, pp. 108-113, 2007.

[71] J. D. Gordan, J. A. Bertout, C. J. Hu, J. A. Diehl, and M. C. Simon, "HIF- $2 \alpha$ promotes hypoxic cell proliferation by enhancing c-Myc transcriptional activity," Cancer Cell, vol. 11, no. 4, pp. 335-347, 2007.

[72] G. Qing and M. C. Simon, "Hypoxia inducible factor-2 $\alpha$ : a critical mediator of aggressive tumor phenotypes," Current Opinion in Genetics \& Development, vol. 19, no. 1, pp. 6066, 2009.

[73] H. Zhang, M. Bosch-Marce, L. A. Shimoda et al., "Mitochondrial autophagy is an HIF-1-dependent adaptive metabolic response to hypoxia," Journal of Biological Chemistry, vol. 283, no. 16, pp. 10892-10903, 2008.

[74] Y. Jang, J. Han, S. J. Kim et al., "Suppression of mitochondrial respiration with auraptene inhibits the progression of renal cell carcinoma: involvement of HIF- $1 \alpha$ degradation," Oncotarget, vol. 6, no. 35, pp. 38127-38138, 2015.

[75] C. V. Dang, J. W. Kim, P. Gao, and J. Yustein, "The interplay between MYC and HIF in cancer," Nature Reviews Cancer, vol. 8, no. 1, pp. 51-56, 2008.

[76] G. L. Semenza, P. H. Roth, H. M. Fang, and G. L. Wang, "Transcriptional regulation of genes encoding glycolytic enzymes by hypoxia-inducible factor 1," Journal of Biological Chemistry, vol. 269, no. 38, pp. 23757-23763, 1994.

[77] J. G. Pastorino, N. Shulga, and J. B. Hoek, "Mitochondrial binding of hexokinase II inhibits Bax-induced cytochrome c release and apoptosis," Journal of Biological Chemistry, vol. 277, no. 9, pp. 7610-7618, 2002.

[78] P. Hydbring and L. G. Larsson, "Tipping the balance: Cdk2 enables Myc to suppress senescence," Cancer Research, vol. 70, no. 17, pp. 6687-6691, 2010.

[79] J. J. Molenaar, M. E. Ebus, D. Geerts et al., "Inactivation of CDK2 is synthetically lethal to MYCN over-expressing cancer cells," Proceedings of the National Academy of Sciences of the United States of America, vol. 106, no. 31, pp. 1296812973, 2009.

[80] S. Campaner, M. Doni, P. Hydbring et al., "Cdk2 suppresses cellular senescence induced by the c-myc oncogene," Nature Cell Biology, vol. 12, no. 1, pp. 54-59, 2010.

[81] B. D. Chang, E. V. Broude, M. Dokmanovic et al., "A senescence-like phenotype distinguishes tumor cells that undergo terminal proliferation arrest after exposure to anticancer agents," Cancer Research, vol. 59, no. 15, pp. 37613767, 1999.

[82] M. Demaria, M. N. O'Leary, J. Chang et al., "Cellular senescence promotes adverse effects of chemotherapy and cancer relapse," Cancer Discovery, vol. 7, no. 2, pp. 165-176, 2017.

[83] J. A. Ewald, J. A. Desotelle, G. Wilding, and D. F. Jarrard, "Therapy-induced senescence in cancer," JNCI: Journal of the National Cancer Institute, vol. 102, no. 20, pp. 15361546, 2010.

[84] M. Milanovic, D. N. Y. Fan, D. Belenki et al., "Senescenceassociated reprogramming promotes cancer stemness," Nature, vol. 553, no. 7686, pp. 96-100, 2018.

[85] T. Reya, A. W. Duncan, L. Ailles et al., "A role for Wnt signalling in self-renewal of haematopoietic stem cells," Nature, vol. 423, no. 6938, pp. 409-414, 2003.

[86] Y. Wang, A. V. Krivtsov, A. U. Sinha et al., "The Wnt/ $\beta$ catenin pathway is required for the development of leukemia stem cells in AML," Science, vol. 327, no. 5973, pp. 16501653, 2010.

[87] S. Capasso, N. Alessio, T. Squillaro et al., "Changes in autophagy, proteasome activity and metabolism to determine a specific signature for acute and chronic senescent mesenchymal stromal cells," Oncotarget, vol. 6, no. 37, pp. 39457-39468, 2015.

[88] H. K. Sanoff, A. M. Deal, J. Krishnamurthy et al., "Effect of cytotoxic chemotherapy on markers of molecular age in patients with breast cancer," JNCI: Journal of the National Cancer Institute, vol. 106, no. 4, article dju057, 2014.

[89] J. Guillon, C. Petit, M. Moreau et al., "Regulation of senescence escape by TSP1 and CD47 following chemotherapy treatment," Cell Death \& Disease, vol. 10, no. 3, p. 199, 2019.

[90] J. R. Whitfield, M.-E. Beaulieu, and L. Soucek, "Strategies to inhibit Myc and their clinical applicability," Frontiers in Cell and Developmental Biology, vol. 5, no. 10, 2017.

[91] S. K. Shukla, A. Dasgupta, K. Mehla et al., "Silibinin-mediated metabolic reprogramming attenuates pancreatic cancerinduced cachexia and tumor growth," Oncotarget, vol. 6, no. 38, pp. 41146-41161, 2015.

[92] D. O. Moon, M. O. Kim, Y. H. Choi, H. G. Lee, N. D. Kim, and G. Y. Kim, "Gossypol suppresses telomerase activity in human leukemia cells via regulating hTERT," FEBS Letters, vol. 582, no. 23-24, pp. 3367-3373, 2008.

[93] R. C. Sun, P. G. Board, and A. C. Blackburn, "Targeting metabolism with arsenic trioxide and dichloroacetate in breast cancer cells," Molecular Cancer, vol. 10, no. 1, p. 142, 2011.

[94] C. C. Huang, S.-Y. Wang, L.-L. Lin et al., "Glycolytic inhibitor 2-deoxyglucose simultaneously targets cancer and endothelial cells to suppress neuroblastoma growth in mice," Disease Models \& Mechanisms, vol. 8, no. 10, pp. 12471254, 2015.

[95] S. Shukla, G. T. MacLennan, C. A. Flask et al., "Blockade of $\beta$ catenin signaling by plant flavonoid apigenin suppresses prostate carcinogenesis in TRAMP mice," Cancer Research, vol. 67, no. 14, pp. 6925-6935, 2007.

[96] E. Gottfried, S. A. Lang, K. Renner et al., "New aspects of an old drug - diclofenac targets MYC and glucose metabolism in tumor cells," PLoS One, vol. 8, no. 7, article e66987, 2013.

[97] M. Momcilovic, S. T. Bailey, J. T. Lee et al., "Targeted inhibition of EGFR and glutaminase induces metabolic crisis in 
EGFR mutant lung cancer," Cell Reports, vol. 18, no. 3, pp. 601-610, 2017.

[98] S. C. Casey, L. Tong, Y. Li et al., "MYC regulates the antitumor immune response through CD47 and PD-L1," Science, vol. 352, no. 6282, pp. 227-231, 2016.

[99] S. Wang, J. Li, J. Xie et al., "Programmed death ligand 1 promotes lymph node metastasis and glucose metabolism in cervical cancer by activating integrin $\beta 4 /$ SNAI1/SIRT3 signaling pathway," Oncogene, vol. 37, no. 30, pp. 4164-4180, 2018.

[100] Y. W. Koh, J.-H. Han, S. Y. Park, D. H. Yoon, C. Suh, and J. Huh, "GLUT1 as a prognostic factor for classical Hodgkin's lymphoma: correlation with PD-L1 and PD-L2 expression," Journal of Pathology and Translational Medicine, vol. 51, no. 2, pp. 152-158, 2017.

[101] E. E. W. Cohen, D. Soulières, C. le Tourneau et al., "Pembrolizumab versus methotrexate, docetaxel, or cetuximab for recurrent or metastatic head-and-neck squamous cell carcinoma (KEYNOTE-040): a randomised, open-label, phase 3 study," The Lancet, vol. 393, no. 10167, pp. 156-167, 2019.

[102] J. Bellmunt, R. de Wit, D. J. Vaughn et al., "Pembrolizumab as second-line therapy for advanced urothelial carcinoma," The New England Journal of Medicine, vol. 376, no. 11, pp. 10151026, 2017.

[103] R. S. Herbst, P. Baas, D. W. Kim et al., "Pembrolizumab versus docetaxel for previously treated, PD-L1-positive, advanced non-small-cell lung cancer (KEYNOTE-010): a randomised controlled trial," The Lancet, vol. 387, no. 10027, pp. 1540-1550, 2016.

[104] A. M. M. Eggermont, C. U. Blank, M. Mandala et al., "Adjuvant pembrolizumab versus placebo in resected stage III melanoma," The New England Journal of Medicine, vol. 378, no. 19, pp. 1789-1801, 2018.

[105] X. Chen, Y. Qian, and S. Wu, “The Warburg effect: evolving interpretations of an established concept," Free Radical \& Biology Medicine, vol. 79, pp. 253-263, 2015. 


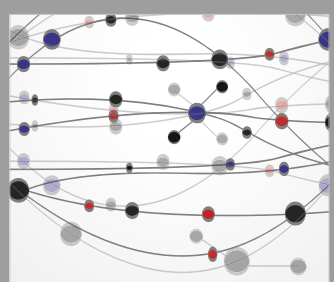

The Scientific World Journal
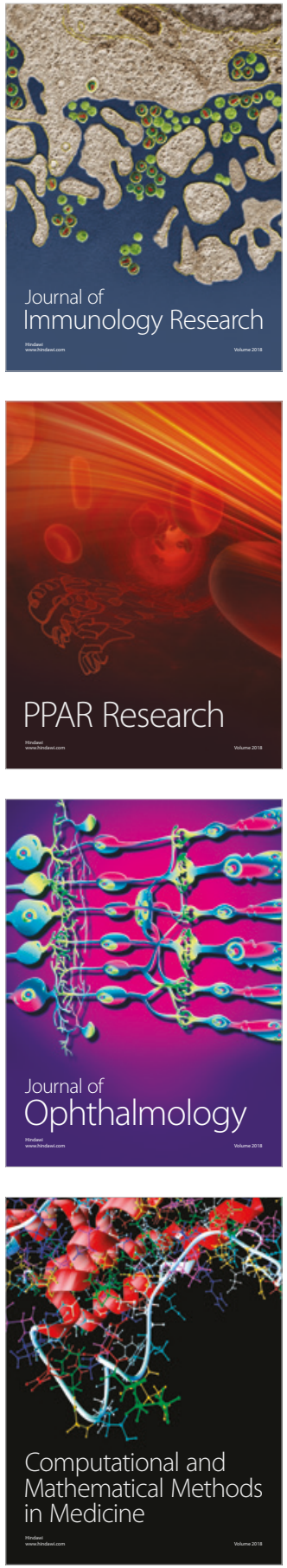

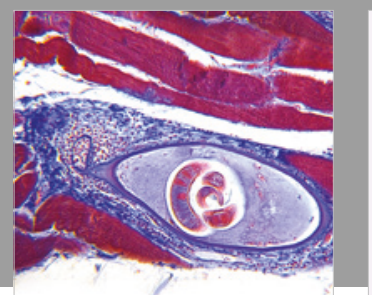

Gastroenterology Research and Practice

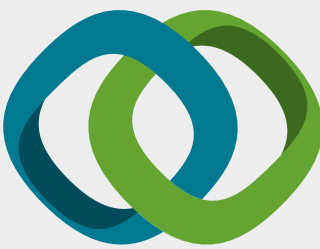

\section{Hindawi}

Submit your manuscripts at

www.hindawi.com
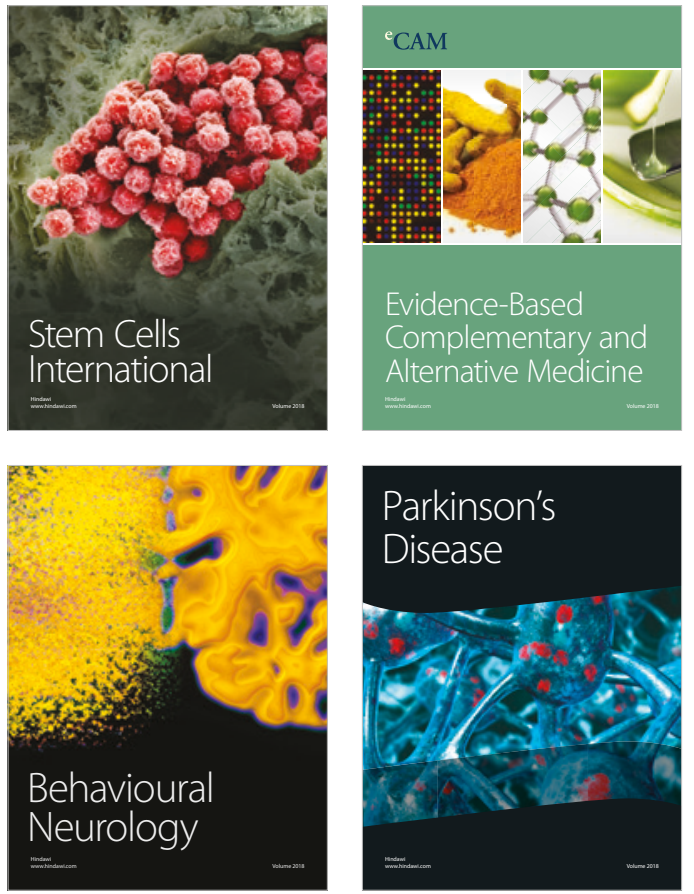

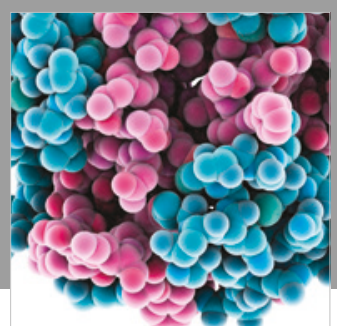

ournal of

Diabetes Research

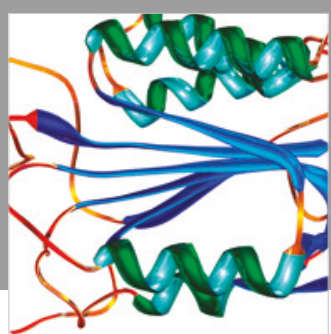

Disease Markers
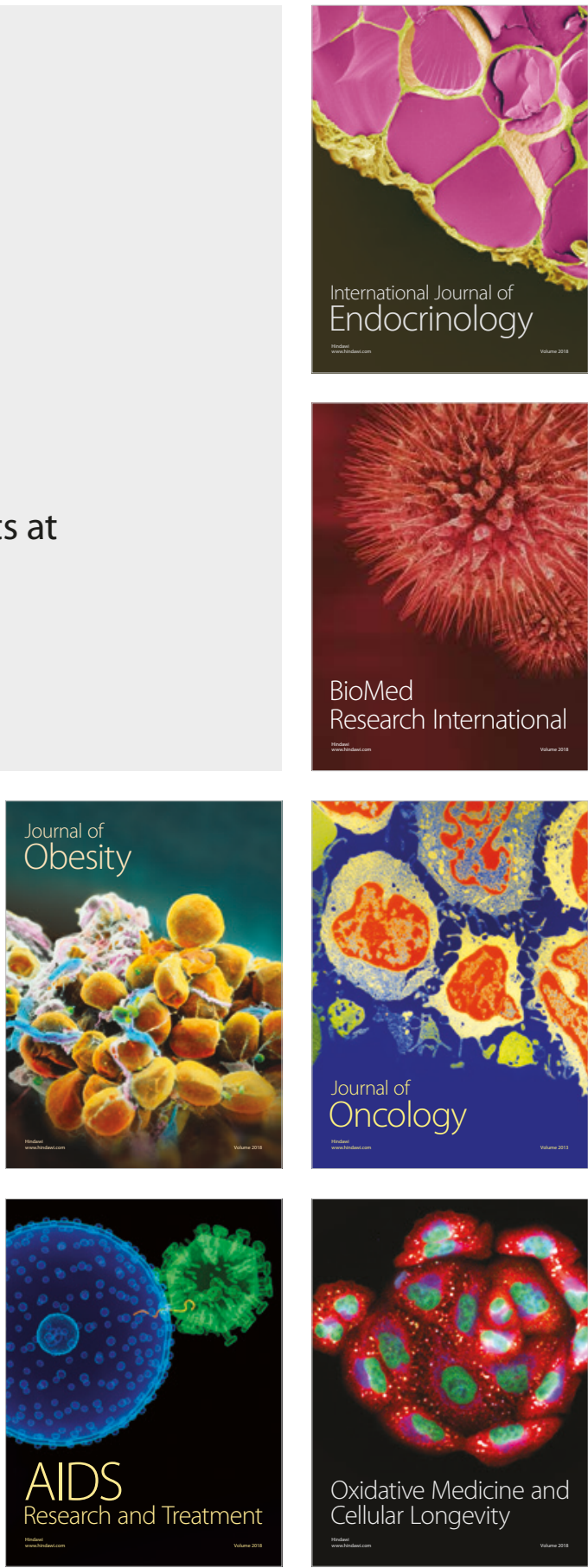